\title{
Histamine, cimetidine and colorectal cancer
}

To the editor - In a News \& Views article in the December issue of Nature Medicine, Morris and Adams reviewed the survival benefit from cimetidine treatment in colorectal cancer ${ }^{1}$. The authors' conclusion that three controlled studies report large survival advantages in cimetidine-treated patients is overstated.

Only one placebo-controlled, randomized trial of cimetidine in colorectal cancer has been published with a total of 192 patients treated after surgery. In their review, Morris and Adams account for a subgroup of these patients (with Dukes' stage $C$ tumors; $n=45$ ) in whom cimetidine tended to prolong survival $(P=$ 0.11 ), but there were no other trends of survival benefit in the cimetidine group, either in patients operated with a curative intent, or in patients with disseminated disease $^{2}$. The authors also reviewed their own data suggesting survival benefit from perioperative treatment with cimetidine in colorectal cancer, but it should be mentioned that this effect was not statistically significant $(P=0.17)$. They also recently presented a randomized trial of cimetidine versus no treatment in 38 patients with metastatic colorectal cancer and found no significant effects of cimetidine on survival - the median survival time for control patients was 588 days as compared with 511 days for cimetidinetreated patients ${ }^{3}$. It seems reasonable to conclude that the benefit of cimetidine in colorectal cancer is uncertain.

Morris and Adams review reports that suggest an association between the occurrence of histamine-containing cells in tumors and poor prognosis, a role for histamine as a paracrine growth factor for tumor cells, and histamine-induced suppression of immune function. Such data would imply that histamine is harmful in cancer, but is it really? First, studies in mice suggest an inverse correlation between the incidence of malignant tumors and tissue histamine, and the incidence of many types of neoplastic disease is reportedly low in conditions associated with excess of histamine such as atopy (reviewed in ref. 4). Secondly, histamine, specifically acting via $\mathrm{H} 2$ receptors, effectively augments natural killer (NK) celldependent destruction of tumor cells in vitro and in vivo ${ }^{5}$. Histamine is particularly effective in activating NK cells when administered together with NK cellactivating cytokines such as interferon- $\alpha$
$(\mathrm{IFN}-\alpha)^{6}$. Indeed, some of the best therapeutic results of IFN therapy in humans have been obtained in polycythemia vera, essential thrombocythemia, mastocytoma, and chronic myelogenous leukemia, all of which are characterized by pronounced hyperhistaminism in peripheral blood'. Third, treatment of tumorbearing animals with histamine delays tumor development and inhibits metastasis (reviewed in ref. 8) and, finally, two recent, uncontrolled studies suggest that histamine may be useful in the treatment of cancer in humans. In metastatic melanoma, histamine (at 20-30 $\mu \mathrm{g}$ per $\mathrm{kg}$ body weight per day) was found to improve response rates and to increase survival time in patients undergoing immunotherapy with interleukin-2 (IL-2) and IFN- $\alpha$ (ref. 9). In the second study, it was reported that histamine synergizes with IL-2 to induce NK cell-mediated destruction of leukemic blasts recovered from patients with acute myelogenous leukemia (AML) in vitro, and that treatment of AML patients in remission with histamine/IL-2 may prolong disease-free survival time ${ }^{10}$.

The activating effects of histamine on NK cells in vitro and on the destruction of NK cell-sensitive tumor cells in vivo are mediated by $\mathrm{H} 2$ receptors $^{5}$; these effects are consequently blocked by $\mathrm{H} 2$ antagonists such as cimetidine. It therefore seems contradictory that cimetidine reduces tumor growth in some rodent cancer models $\mathrm{s}^{1,11}$. One of several possible explanations may relate to the fact that histamine, acting via $\mathrm{H} 2$ receptors, is an NK-cell activator but a suppressor of Tcell function, and it is conceivable that effects of cimetidine or histamine may be determined by the sensitivity of tumor cells to NK- and T cell-dependent defense mechanisms.

Morris and Adams advocate a broader use of cimetidine in cancer including melanoma, but it should be mentioned that there are many reports suggesting that cimetidine is ineffective in human melanoma as monotherapy ${ }^{12}$, as well as when administered together with immunoactivators ${ }^{13}$.

Kristoffer Hellstrand, Mats Brune, Ulf-Henrik MEllqvist \&

PETER NAREDI

Departments of Virology, Haematology, and Surgery, University of Göteborg, Sweden
Morris replies - The central issue of the effect of adjuvant cimetidine on survival in colorectal cancer is far from resolved, hence the question mark in the title of our article. In each of the randomized controlled studies we quoted, a survival benefit was seen. As patients numbers were low, statistical significance was achieved in only one, and in one the effect was restricted to state $\mathrm{C}$ cancers. Although this is hardly conclusive, it is certainly of interest.

Hellstrand et al., when commenting on our recently reported pilot comparison of cimetidine/5FU versus $5 \mathrm{FU}$ alone ${ }^{13}$, omit to mention that a significantly higher objective response rate [in levels of secreted carcinoembryonic antigen] was seen in the cimetidine/5FU group $(P=0.023)$. We believe, however, that this is likely to be related to a direct effect of cimetidine on 5FU metabolism and not relevant to the discussion on the role of adjuvant cimetidine in early stage cancer. In early stages there is a small volume of disease present in patients with an active immune system. In advanced disease neither of these conditions are present and immunostimulatory treatments have little effect.

The effect of histamine on cancer incidence is interesting, but again not directly relevant to this discussion. Indeed, there are very good data from postmarketing studies in nearly 27,000 patients that cimetidine does not reduce the incidence of colorectal cancer ${ }^{14}$, but this does not discount its having an effect on cancers once established.

The effect of histamine itself on cancer growth is complex and does not necessarily imply an opposite effect of histamine antagonists. As Hellstrand et al. discuss, both histamine and cimetidine have been demonstrated to stimulate NK cell function. Clinical stud. ies have found cimetidine to enhance NK cell function in cancer patients ${ }^{15}$. Histamine acts on cancer growth through a variety of means including, but probably not restricted to, immunological and microvascular mechanisms, and this has led to contradictory experimental results. Although other studies have found histamine to inhibit cancer growth, these have been in other tumor types. Our own work in human colorectal cancer cell lines has shown that histamine stimulates cAMP release, in vitro and in vivo ${ }^{16}$. Although we believe that it is established 